\title{
An Secure Hierarchical Key Agreement Scheme for Wireless Sensor Networks
}

\author{
Kefei Mao*, Jie Chen and Jianwei Liu \\ School of Electronic and Information Engineering, Beihang University, \\ Beijing, China \\ *Corresponding Author: Kefei Mao. E-mail:owen.buaa@gmail.com
}

\begin{abstract}
In Wireless Sensor Networks (WSN), a key agreement scheme is an essential task for secure communications. Recently, Lee and Kim proposed a hierarchical key agreement scheme for the fresh key establishment in WSN. This scheme achieves a secure session key agreement. In this paper, we analyze the security of the scheme and illustrate that their scheme is unconfident against the insider attack in practice. Moreover, it is also difficult to resist the replay attack in this scheme. Then, we proposed novel scheme inspired by their scheme. The entities of our scheme include a Sinkbesides the sensor nodes, which interrupts the construction of the insider attack. Our scheme adopts the timestamp mechanism to resist replay attack, which could decrease the storing requirement of the sensor nodes. Thus, it is more practical and realistic. We illustrate that our proposal can provide stronger security than Lee and Kim'scheme.
\end{abstract}

Keywords: Security, Key Agreemenn Scheme, Wireless Sensor Networks (WSN), Authentication

\section{Introduction}

The more wireless sensor technology is being developed, the more benefits are brought to civilian and military requirements by using the Wireless Sensor Networks (WSN). WSN has become a network of integrated sensor technologies, and supports multiple extensible applications, such as emergency response, medical monitoring and battlefield management [1 35. At the same time, WSN has also attracted various attacks due to the significance of its data 4 . Therefore, how to make the session key agreement securely and efficiently between any two leaf nodes in the open networks becomes a primary security issue [5-7]. The essence of the problem is to implement a hierarchical key agreement schenne [8-10].

In recent years, many security schemes have been proposed for WSN likely environments [6]. In the early days, the public key cryptography (PKC) is the primary solution in WSN as well as other cryptography system [11-14]. Due to the reliability and the credibility of the traditional pair-wise key establishment techniques, the methods are widely cognitive. In particular, the schemes were proposed by using elliptic curve cryptography (ECC) because of the storage and computing cost advantages [15-17]. With the development of identity-based cryptography (IBC) [18] and applications [19, 20], some papers [21-23] have used IBC and pairing-based cryptography for key distribution in WSN.

Recently, Inspired by the Guo et al.'s research [8], Kim proposed a hierarchical key agreement protocol applicable to WSN [24]. The scheme is also based on IBC, and claims that it resists the corruption of any sensor nodes in the pyramid. Unfortunately, Lee and Kim found that the scheme in [24] fail to achieve freshness of the session key. Also, Lee and Kim proposed an improved scheme to satisfy the freshness by using the nonce [7] and inherit the security advantages from the paper [24]. 
In this paper, we show that Lee and Kim's scheme [7] failed to resist the replay attack and the insider attack in practice. We start from constructing a realistic security model. Then, we analyze that the scheme is vulnerable to the replay attack in our security model because the security assumption is too hard to achieve in practice. Moreover, the scheme is hard to resist the insider attack. An adversary, as a cluster head $(\mathrm{CH})$ or a cluster member (CM), could legally acquire the private key, and launch an attack, successfully. In order to resist the attacks, we propose a novel hierarchical key agreement scheme in WSN. Our scheme keeps the quality of key freshness in Lee and Kim's scheme and overcomes the weaknesses of the scheme in our security model.

The remainder of this paper is organized as follows. Section 2 gives the problem characteristics and notations. Section 3 briefly reviews the Lee and Kim's scheme. Section 4 illustrates the drawbacks of their scheme in our security model. Section 5 provides a novel scheme. Section 6 gives a security and performance analysis of our proposed scheme. Finally, the conclusion is presented in Section 7.

\section{Preliminaries}

In this section, we describe the basic system model in WSN and the mathematical backgrounds in our paper. Basic notations are provided at the end of the section.

\subsection{Basic System Model}

A typical WSN configuration involves three parties [25], namely a Sink, the Cluster Heads (CH), and the Cluster Members (CN). We assume there are $n \mathrm{CHs}$, namely $\left\{\mathrm{CH}_{i}\right\}_{i=1}^{n}$. Every CH whose identity is $\left\{\mathrm{CH}_{i}\right\}_{i=1}$ manages ${ }^{m} \mathrm{CMs}$, namely $\left\{\mathrm{CM}_{i j}\right\}_{j=1}^{m}$. All $\mathrm{CMs}$ communicate with others through their CH and the Sink. Figure 1 illustrates the basic system model of the WSN.

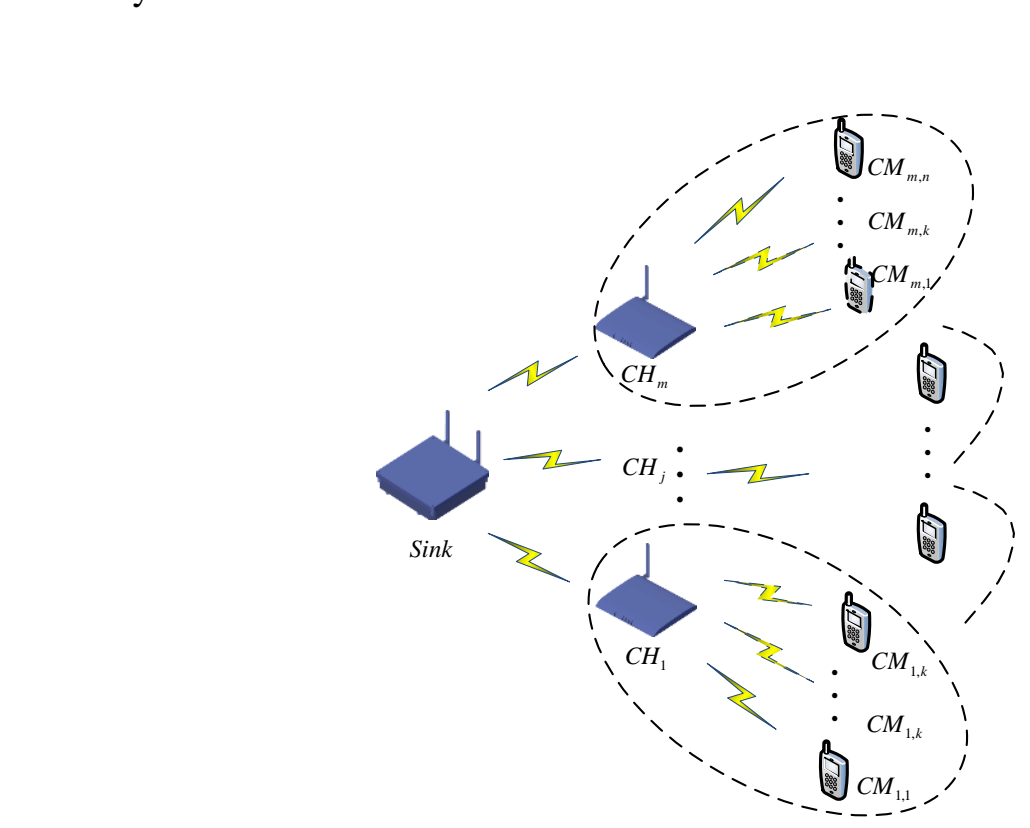

Figure 1. Basic System Model of WSN 


\subsection{Mathematical Backgrounds}

\subsubsection{Bilinear Maps}

Let $G_{1}$ and $G_{2}$ be two cyclic groups of prime order $p$, and Let $P$ be a generator of $G_{1}$. The bilinear pairing is a map $\hat{e}: G_{1} \times G_{1} \rightarrow G_{2}$ with following properties.

Bilinearity. For all $P, Q \in G_{1}$ and $a, b \in Z_{q}^{*}, \hat{e}(a P, b Q)=\hat{e}(P, Q)^{a b}$.

Non-degeneracy. $\hat{e}(P, P) \neq 1$.

Computability. For all $P, Q \in G_{1}$, we can find an efficient algorithm to compute $\hat{e}(P, Q)$

\subsubsection{Discrete Logarithm Problem}

Given a randomly chosen $h=a P$ and $P$ in an additive cyclic group $Q 1$, with $a \in Z_{q}{ }^{*}$, compute $a \in Z_{q}{ }^{*}$. We say that the ${ }^{(t-\varepsilon)}$-DLP assumption holds in $G_{1}$ if on ttime algorithm has non-negligible advantage $\varepsilon$ in solving the Discrete Logarithm Problem (DLP) in $G_{1}$

\subsection{Notations}

To provide a quick reference, the basic notations used in this paper are listed in Table 1 .

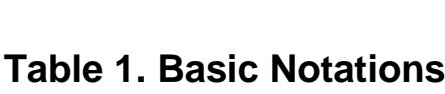

\begin{tabular}{l|l}
\hline \multicolumn{1}{c|}{ Notations } & Descriptions \\
\hline$C H_{i}$ & The cluster head $i$ \\
$C M_{i j}$ & The cyster member node $j$ in the cluster head $i$ \\
$I D_{i}$ & The identity of $i$ \\
$A D_{i}$ & The amplified identity of $i$ \\
$H_{1}(\cdot)$ & The hash function, which maps $\{0,1\}^{*} \rightarrow G_{1}$ \\
$H_{2}(\cdot)$ & The hash function, which maps $G_{2} \times G_{1}{ }^{2} \times\{0,1\}^{n} \rightarrow\{0,1\}^{n}$ \\
$H_{3}(\cdot)$ & The hash function, which maps $G_{1}{ }^{4} \times\{0,1\}^{2 n} \rightarrow\{0,1\}^{n}$ \\
$T_{i}$ & The $i-t h$ timestamp \\
\hline
\end{tabular}

\section{Review of the Scheme in Paper}

In this section, we briefly review the Lee and Kim's freshness consideration key agreement scheme in paper [7], which consist of two phases: Hierarchical Key Settlement Phase, Session Key Agreement and Secure Communication Phase. It is assumed that each entities shares two groups $G_{1}$ and $G_{2}$ of prime order $p$ with a bilinear map $\hat{e}: G_{1} \times G_{1} \rightarrow G_{2}$ and a cryptographic hash function $H:\{0,1\}^{*} \rightarrow G_{1}$. The basic transmission of the scheme is shown in Figure 2.

\subsection{Hierarchical Key Settlement Phase}

Step K1. Sink picks three random numbers $S_{1}, s_{2}, s_{3} \leftarrow Z_{q}^{*}$ as the master private keys. Then, Sink computes an amplified identity $A D s=H(I D s)$ and a public key $s_{1} A D s$, 
where $I D s$ is the real identity of Sink. Then, Sink secure stores the master private key $\left(s_{1}, s_{2}, s_{3}\right)$ and the amplified identity $A D_{s}$. Finally, Sink sends the data package $\left\{\left(s_{1} A D_{s}, s_{2}, s_{3}\right), A D_{s}\right\}$ to the $\mathrm{CH}$ via a secure way.

Step K2. After $\mathrm{CH}\left({ } D_{\mathrm{CH}_{i}}\right)$ receives the package, it computes $A D_{C H_{i}}=H\left(I D_{C H}\right)$ and $S_{2} A D_{C H_{i}}$. Then, $\mathrm{CH}$ secure stores the private key $\left(S_{1} A D_{S}, S_{2}, S_{3}\right)$ and the amplified identities $\left(A D_{s}, A D_{C H}\right)$. Finally, $\mathrm{CH}$ sends the data package $\left\{\left(S_{1} A D_{s}, S_{2} A D_{C H i}, S_{3}\right), A D_{s}, A D_{C H_{i}}\right\}$ to its member nodes $\left\{C M_{i j}\right\}_{j=1}^{m}$ via a secure way.

Step K3. After the CM ( $\left.I D_{C M_{i j}}\right)$ receives the package, it computes $A D_{C M_{i j}}=H\left(I D_{C M_{i j}}\right)$ and $s_{3} A D_{C M_{i j}}$. Then, $C M$ secure stores the private keys $\left(s_{1} A D_{s}, S_{2} A D_{C H_{i}}, S_{3} A D_{C M_{i j}}\right)$ and the amplified identity $\left(A D_{s}, A D_{C H}, A D_{C M_{i j}}\right)$, respectively.

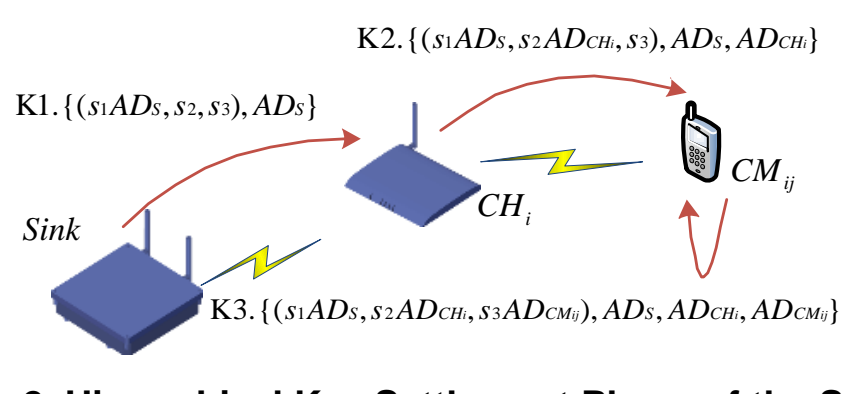

Figure 2. Hierarchical Key Settlement Phase of the Scheme

\subsection{Session Key Agreement and Secure Communication Phase}

Two CMs ( $C M_{i j}$ and $C M_{k l}$ ) compute a session key as follows.

Step C1. The CM ( $C M D$ ) chooses a random number $r_{1}$, and computes $R_{1}=r_{1} A D_{C M_{i j}}$. The session key $s k$ is given by the following formula. Here $A D_{s^{\prime}}=H(I D s)$, $A D_{C H k^{\prime}}=H\left(I D_{C H}\right)$ and $A D_{C M M^{\prime}}=H\left(I D_{C M k}\right)$.

$$
s_{k}=\hat{e}\left(s_{1} A D_{s}, A D_{S^{\prime}}\right) \cdot \hat{e}\left(s_{2} A D_{C H_{i}}, A D_{C H_{k}}{ }^{\prime}\right) \cdot \hat{e}\left(s_{3} A D_{C M_{i j}}, A D_{C M_{k l}}\right)^{r_{1}}
$$

Then, $C M_{i l}$ computes the verifier $V_{1}=H\left(s k, R_{1}\right)$, and sends the data package $\left\{R_{1}, V_{1}\right\}$ to the other $\mathrm{CM}$ ( $C M$ P

Step C2. After $C M_{k l}$ receives the package, it computes the session key $s k$ by the following formula, where $A D_{S^{\prime}}=H\left(I D_{S}\right)$ and $A D_{C H_{i}^{\prime}}=H\left(I D_{C H_{i}}\right)$.

$$
s k^{*}=\hat{e}\left(s_{1} A D_{s}, A D_{s^{\prime}}\right) \cdot \hat{e}\left(s_{2} A D_{C H_{k}}, A D_{C H_{i}}\right) \cdot \hat{e}\left(s_{3} A D_{C M_{k l}}, R_{1}\right)
$$

Then, $C M_{k l}$ also computers the verifier $V_{1}{ }^{*}=H\left(s k^{*}, R_{1}\right)$. Only if $V_{1}{ }^{*}$ is equal to $V_{1}$, CM. assures the correctness of $s k^{*}$.

Step C3. After $C M_{k l}$ assures the session key $s k^{*}$, it encrypts the plain data DATA to get the encrypt data EDATA by using the key. Then, $C M_{k l}$ computes the verifier $V_{2}=H\left(s k^{*}, E D A T A\right)$, and sends the data package $\left\{E D A T A, V_{2}\right\}$ to $C M_{i j}$.

Step C4. After $C M_{i j}$ receives the package, it also computes the verifier $V_{2}{ }^{*}=H(s k, E D A T A)$. Only if $V_{2}^{*}$ is equal to $V_{2}, C M_{i j}$ assures the correctness of the encrypt data EDATA and the session key $s k$. 


\section{Cryptanalysis of Lee and Kim's Scheme}

In this section, we propose a more practical security model. Moreover, we point out Lee and Kim's scheme [7] suffers two attacks in our model. The details are explained in the following section.

\subsection{Our Security Model}

The authors of the paper proposed a hierarchical key agreement scheme in WSN. The authors assume the insider's secret values are reliable and security, such as the private key of the $\mathrm{CHs}$ and the CMs. The adversary can only implement the active and passive attack by controlling the insecurity channel. However, it is more useful to carefully consider the corruption risk of the internal nodes. In practice, the WSN is easily attacked from inside. For example, the adversary can capture some nodes, and then use the side channel attacks to get the secret values of the nodes. Therefore, we propose a more practical security model.

Inspired by the papers, we enhance the ability of the adversary in our model. Same as others, the adversary can totally control over the communication channel More precisely, the adversary may eavesdrop, intercept, modify, replay or inject the communication between any entities in the WSN (e.g. the channel between $C M$ and $C M_{k l}$ ). Furthermore, the adversary can also corrupt some the secret parameters from the $\mathrm{CHs}$ and the CMs except those of the entities who are attacking by the adversary. This state imitates that the insider is corrupted in the WSN. Under above conditions, the scheme should have the ability to resist various kinds of attacks, and achieve the following security goals. (1) Key security. Any adversary cannot obtain the current session key. (2) Known-key security. Any adversary compromised a sared session key cannot obtain the current session key.

\subsection{Weaknesses}

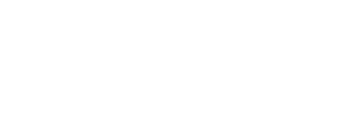

According to Lee and Kim's paper [7], we find that the scheme has the following disadvantages in our security model.

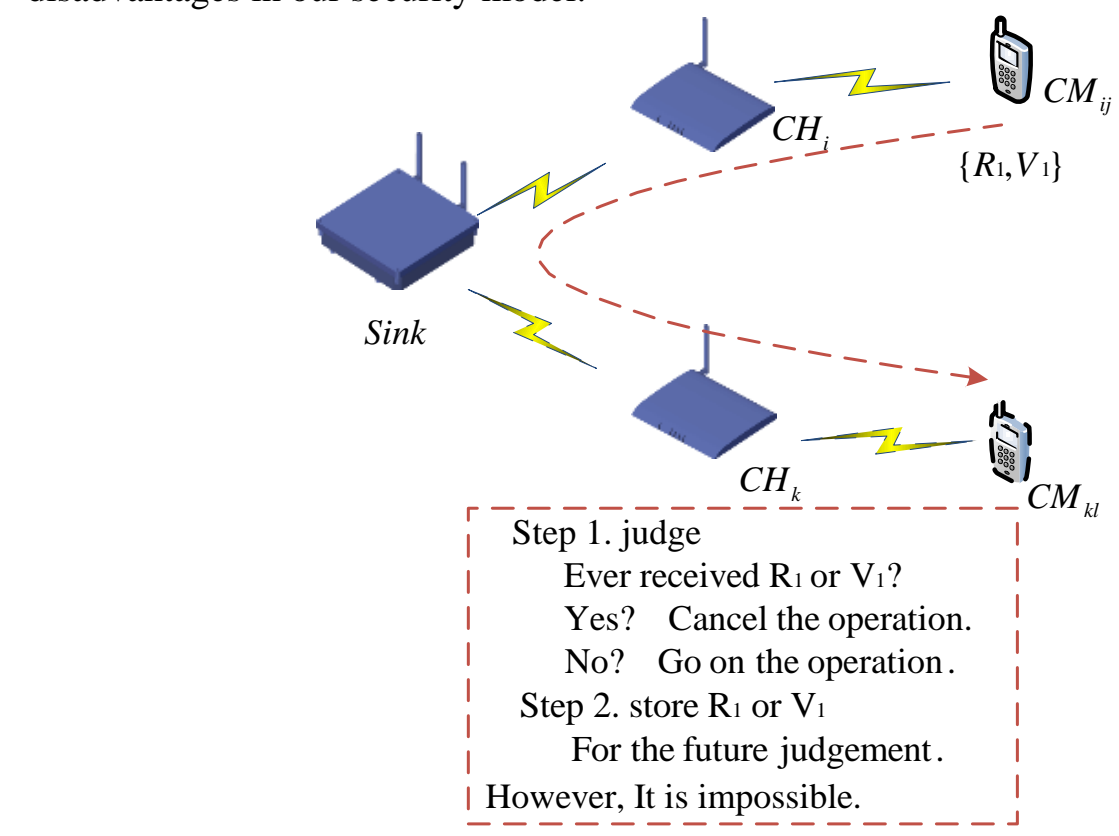

Figure 3. Cannot Resist the Replay Attack 


\subsubsection{It Cannot Resist the Replay Attack}

The scheme will has security problems if the adversary launches the replay attack in real world. As we know, the nonce $R_{1}$ is used to prevent the replay attack in the paper. However, the scheme cannot resist replay attack in practice. More precisely, it is essential that the CM should judge whether the nonce $R_{1}$ and verifier $V_{1}$ have ever received. In order to make a judgment, the CMs should store the received nonce $R_{1}$, interminably. However, it is obviously impossible to sensor nodes because of the limited resources. Thus, it cannot resist the replay attack to use the method in Lee and Kim's scheme. The more details are described as follows.

As is shown in Figure 3, when the adversary has captured the data packet $\left\{R_{1}, V_{1}\right\}$ from $C M_{i j}$ to $C M_{k l}$, he/she can store it. Then he/she can replay the nonce $R_{1}$ and the verifier $V_{1}$ whenever he/she wants. If the victim CM (CMkl $)$ receives the data packet $\left\{R_{1} V_{1}\right\}$, it is obviously that it can believe the package from the real node $C M_{i j}$ because $V$ Is equal to $H\left(s k^{*}, R_{1}\right)$. In order to avoid the attack, $C M_{k l}$ should be stored the nonce $R_{1}$ received before and judge whether the current nonce $R_{1}$ is a replay attack. However, there is no enough resource to support the storing and querying nonce in CM. Thus, Lee and Kim's scheme cannot resist the replay attack in practice.

\subsubsection{It Cannot Resist the Insider Attack (the Cluster Head Disguise)}

We now demonstrate that the Lee and Kim scheme is vulnerable to the one kind of insider attack. The adversary who has registered as a legal $\mathrm{CH}\left(\mathrm{CH}_{A}\right)$ as shown in Figure 4. According to the definition of ousecurity model, the adversary can intercept the communication data in the WSN because the wireless channel is openness. The adversary who disguises a $\mathrm{CH}$ successfully attacks a communication between $C M_{i j}$ and $C M_{k l}$ as follows.

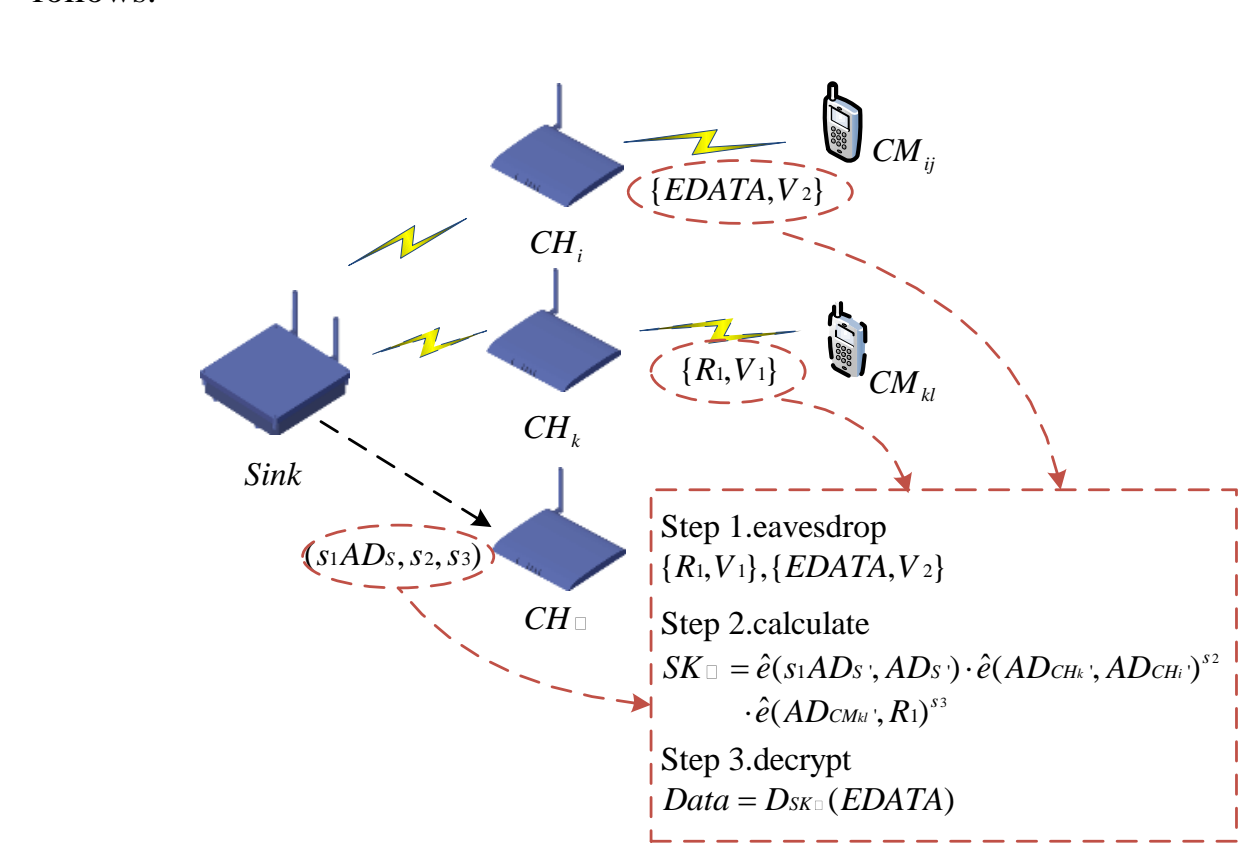

Figure 4. Cannot Against Insider Attack 
Step H1. Assume that $\mathrm{CH}_{A}$ is an adversary who has registered as a $\mathrm{CH}$, and then it can legally receive a private key set $\left(s_{1} A D_{s}, s_{2}, s_{3}\right)$ from the Sink (Step K1).

Step H2. Suppose $C M_{i j}$ and $C M_{k l}$ are victim $C M$ s who send the data packages through their $\mathrm{CHs}$ ( $\mathrm{CH}_{i}$ and $\mathrm{CH}_{k}$ ) and Sink. When $\mathrm{CM}_{i j}$ runs the Step C1, the adversary can intercept the data package $\left\{R_{1}, V_{1}\right\}$ because channel is unsecure between $C M_{i j}$ and $C M_{k l}$.

Step H3. When $C M_{k l}$ sends back the encrypt data EDATA to $C M_{i j}$ at the Step C3, the adversary can also intercept the data package $\left\{E D A T A, V_{2}\right\}$.

Step H4. After the above steps, the adversary $\mathrm{CH}_{A}$ can compute the key $s k_{A}$ by the following formula. Here, the values $A D_{S^{\prime}}=H\left(I D_{S}\right), A D_{C k^{\prime}}=H\left(I D_{C H_{k}}\right)$, $A D_{C H_{i}}=H\left(I D_{C H i}\right)$ and $A D_{C M k l^{\prime}}=H\left(I D_{C M k}\right)$

$$
\begin{aligned}
s k_{A} & =\hat{e}\left(s_{1} A D_{s}, A D_{S^{\prime}}\right) \cdot \hat{e}\left(A D_{C H^{\prime}}, A D_{C H_{i}}\right)^{s 2} \cdot \hat{e}\left(A_{\left.C_{C M k^{\prime}}, R_{1}\right)^{s 3}}\right. \\
& =\hat{e}\left(s_{1} A D_{s}, A D_{S^{\prime}}\right) \cdot \hat{e}\left(s_{2} A D_{C H_{k}}, A D_{C H_{i}}\right) \cdot \hat{e}\left(s_{3} A D_{C M_{k}}, R_{1}\right) \\
& =s k
\end{aligned}
$$

Step H5. The adversary $\mathrm{CH}_{A}$ can decrypt EDATA to obtain the encrypt data between $C M_{i j}$ and $C M_{k l}$ by using the session key $s k_{A}$ because the keys $s k^{*}$, $s k$ and $s k^{*}$ are equal. Thus, Lee and Kim's scheme [7] cannot resist the insider attack when the adversary disguises a $\mathrm{CH}$.

\subsubsection{It Cannot Resist the Insider Attack (the Clus er Member Disguise)}

Moreover, there is another insider attack as follows. The adversary who has registered as a legal $\mathrm{CM}$ ( $\mathrm{CM}_{k A}$ ) as shown in Figure 5. The adversary has the same $\mathrm{CH}$ ( $\mathrm{CH}_{k}$ ) with the one victim $C M_{k l}$. Therefore, the adversary is a neighbor node of the victim. The adversary launches an attack to the communication between $C M_{i j}$ and $C M_{k l}$ as follows.

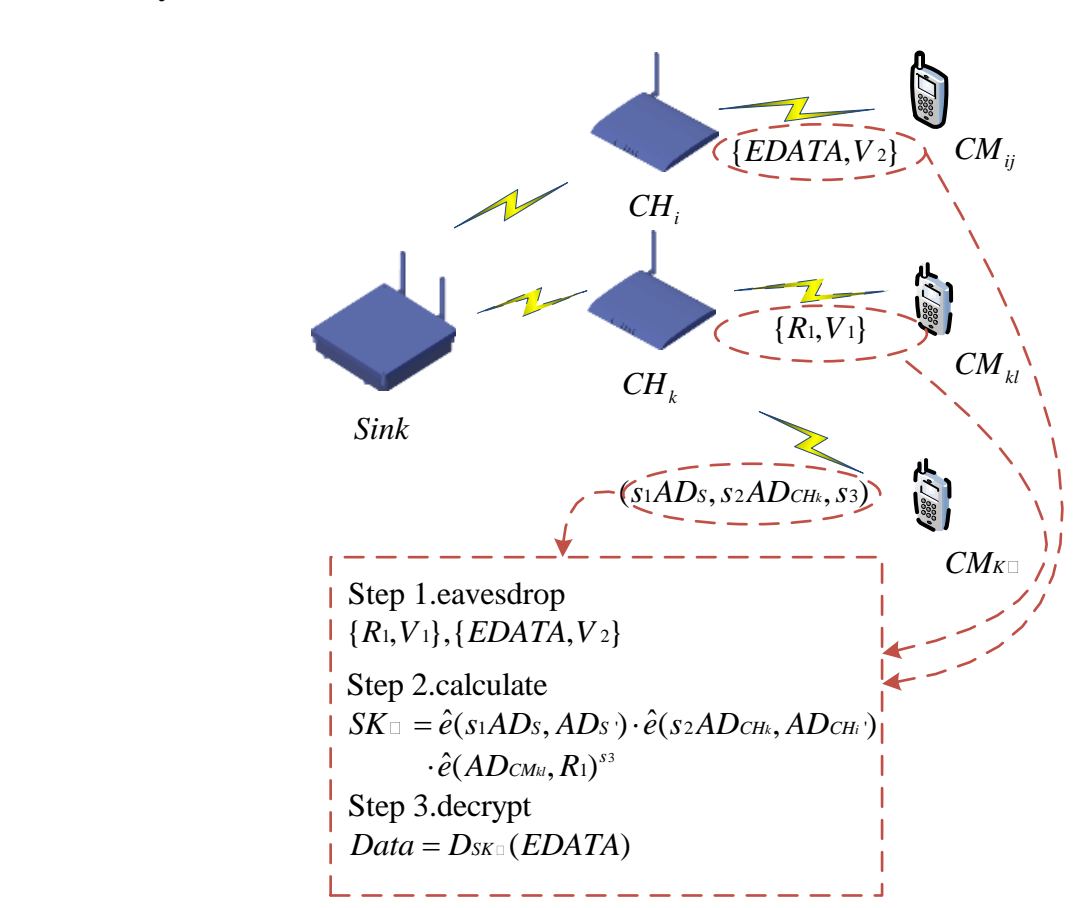

Figure 5. Cannot Against Insider Attack 
Step M1. Assume that $\mathrm{CH}_{A}$ is an adversary who has registered as a $\mathrm{CM}$ in a $\mathrm{CH}$ $\left(\mathrm{CH}_{k}\right)$, and then it legally receives a private key set $\left(\mathrm{s}_{1} \mathrm{AD} \mathrm{D}_{s}, \mathrm{~S}_{2} \mathrm{AD} \mathrm{DH}_{\mathrm{C}}, \mathrm{S}_{3}\right)$ from $\mathrm{CH}_{k}$ (Step K2).

Step M2. Suppose two CMs ( $C M_{i j}$ and $C M_{k l}$ ) are victim nodes. It is same above describe that the adversary can intercept the data $\left\{R_{1}, V_{1}\right\}$ and $\left\{E D A T A, V_{2}\right\}$.

Step M3. After the above steps, The adversary $\mathrm{CH}_{A}$ can compute the session key as follows. Here, the values $A D_{S^{\prime}}=H\left(I D_{S}\right), A D_{C i_{i}}=H\left(I D_{C H_{i}}\right)$ and $A D_{C M_{k \prime}^{\prime}}=H\left(I D_{C M_{k l}}\right)$.

$$
\begin{aligned}
s k_{A} & =\hat{e}\left(s_{1} A D_{s}, A D_{S^{\prime}}\right) \cdot \hat{e}\left(s_{2} A D_{C H_{k}}, A D_{C H_{i}}\right) \cdot \hat{e}\left(A D_{C M_{k l}}, R_{1}\right)^{s 3} \\
& =\hat{e}\left(s_{1} A D_{s}, A D_{S^{\prime}}\right) \cdot \hat{e}\left(s_{2} A D_{C H_{k}}, A D_{C H_{i}}\right) \cdot \hat{e}\left(s_{3} A D_{C M_{k}}, R_{1}\right) \\
& =s k
\end{aligned}
$$

Step M4. It is obviously that the keys $s k_{A}, s k$ and $s k^{*}$ are equal. Therefore the adversary $\mathrm{CH}_{A}$ can decrypt EDATA to obtain the plain text DATA using the key $\mathcal{K}_{A}$. Thus, Lee and Kim's scheme cannot resist the insider attack when the adversary disguises a neighbor $\mathrm{CM}$ with one of two victims.

\section{Our Enhanced Scheme}

In this section, we propose an improved scheme based on identity based cryptography, which can overcome the weaknesses of Lee and Kim's scheme in Section 4. Our scheme construction is inspired by the papers [7-8, 190. Our schême consists of three operational phase: System Parameter Generation Phase, Initial Phase, Authentication and Transmission Phase. The details of our scheme are described as follows.

\subsection{System Parameter Generation Phase}

Similar to the papers [7-8], we use the identity-based encryption (IBE) and the hierarchical structure in our scheme. More specifically, the identity of a sensor node is his/her public key, and his ber private key generates by the Private Key Generator (PKG) in Sink. The parent nodes calculate and release the private key set for their descendants.

Step G1. Sink generates two addition groups $G_{1}$ and $G_{2}$ of prime order $p$ with a bilinear map $\hat{e} \cdot G_{1} \times G_{1} \rightarrow G_{2}$. Then, Sink chooses three hash functions satisfied $H_{1}:\{0,1\}^{*} \rightarrow G, H_{2}: G_{2} \times\left\{\times\{1\}^{n} \rightarrow\{0,1\}^{n}\right.$ and $H_{3}: G_{1}{ }^{4} \times\{0,1\}^{2 n} \rightarrow\{0,1\}^{n}$. After that, it randomly chooses $-m$ random numbers $\left\{s_{1}, s_{2}, s_{3}, s r_{i} \leftarrow Z_{q}^{*} \mid i \in(1, \cdots, m)\right\}$ as a master key of Sink and a random generator $P_{0} \in G_{1}$. Here, $m$ is the number of CHs in WSN. Finally, Sink secure stores the master key $M K=\left\{s_{1}, s_{2}, s_{3}, s r_{i} \leftarrow Z_{q}^{*} \mid i \in(1, \cdots, m)\right\}$ and publishes the public parameters params $=\left\{q, G_{1}, G_{2}, P_{0}, \hat{e}, H_{1}, H_{2}, H_{3}, s_{1} P_{0}, s_{2} P_{0}, s_{3} P_{0}, s r_{i} P_{0} \mid i \in(1, \cdots, m)\right\}$.
5.2. Initial Phase

Step I1. When a $\mathrm{CH}\left(\mathrm{CH}_{i}\right)$ with identity ${ }^{I} \mathrm{CH}_{\mathrm{CH}_{i}}$ wants to register in WSN. It sends his identity $I D_{C H_{i}}$ to Sink. Sink computes the amplified identities $\left(A D_{s}, A D_{C H_{i}}\right)$ and the private key $\left(s_{1} s r_{i} A D_{s}, s_{2} s r_{i} A D_{C H}, S_{3} S r_{i}\right)$.Here, the values $A D_{s}=H_{1}\left(I D_{s}\right)$ and $A D_{C H_{i}}=H_{1}\left(I D_{C H_{i}}\right)$. Then, the Sink sends the data package $\left\{\left(s_{1} S r_{i} A D_{s}, S_{2} S r_{i} A D_{C H_{i}}, S_{3} S r_{i}\right),\left(A D_{s}, A D_{C H_{i}}\right)\right\}$ to $C H_{i}$ via a secure channel. Finally, $C H_{i}$ keeps the received information in its secure memory. 
Step I2. When a $\mathrm{CM}\left({ }^{C M_{i j}}\right)$ with identity $I D_{C H_{i}}$ wants to register in WSN. It sends his identity $I D_{C M_{i j}}$ to its $\mathrm{CH}\left(C_{i}\right)$. $C_{i}$ computes $A D_{C M_{i j}}=H\left(I D_{C M_{i j}}\right)$ and $S 3 S r_{i} A D_{C M_{i j}}$. Then, the $\mathrm{CH}\left(\mathrm{CH}_{i}\right)$ sends the private key set $\left\{\left(s_{1} S r_{i} A D_{s}, S_{3} S r_{i} A D_{C M_{i j}}\right),\left(A D_{s}, A D_{C M_{i j}}\right)\right\}$ to its $C M\left(C M_{i j}\right)$, securely. Finally, $C M_{i j}$ keeps the received information in its secure memory.

\subsection{Authentication and Key Agreement Phase}

When $C M_{i j}$ wants to establish a session key $s k$ with $C M_{k l}$ by the helping of Sink, the following steps are executed among $C M_{i j}, C M_{k l}$ and Sink in Figure 6.

Step A1. $C M_{i j}$ chooses a random number $r_{1}$. Then it computes an amplified identity $A D_{C M_{k l}}=H_{1}\left(I D_{C M_{k}}\right), R_{1}=r_{1} A D_{C M_{i j}}$ and $R_{s}=r_{1} A D_{\mathrm{s}}$. The temporary key $k$ is calculated by using the above parameters and the private key set $\left(S_{1} S r_{i} A D_{s}, S_{3} S r_{i} A D_{C M_{i j}}\right)$

Then, $C M_{i j} \quad$ computes the values $k=\hat{e}\left(s_{1} S r_{i} A D s, A D_{s}\right)^{r_{1}} \cdot \hat{e}\left(s_{3} S r_{i} A D_{C M_{i j}}, A D_{C M_{k}}\right)^{r_{1}}$

$V_{2}=H_{2}\left(s_{1} S r_{i} A D_{s}, S_{3} S r_{i} A D_{C M_{i j}}, R_{1}, R s, T_{1}, V_{1}\right)$ and $V_{1}=H_{2}\left(s k, A D_{\left.C M_{i j}, A D_{C M}, " 0 "\right)}\right.$.Then it sends $M_{1}=\left\{A D_{C M_{k l}}, T_{1}, R_{1}, R_{s}, V_{1}, V_{2}\right\}$ to Sink. Here, and $T_{1}$ is a timestamp.

Step A2. After received the data package $M A$, Sink verifies the timestamp $T_{1}$ whether it is within the valid time for communication. If it is invalid, the program terminates. Otherwise, Sink judges whether the regeived $V_{2}$ is equal to $H_{2}\left(s_{1} S r_{i} A D_{s}, S_{3} S r_{i} A D_{C M_{i j}}, R_{1}, R_{s}, T_{1}, V_{d}\right.$ and computes whether $\hat{e}\left(R_{s}, A D_{C M_{i j}}\right)=\hat{e}\left(R_{1}, A D_{s}\right)$.

Only if they are all equal, Sink Convince the two values $R_{1}$ and $R_{2}$, then it computes

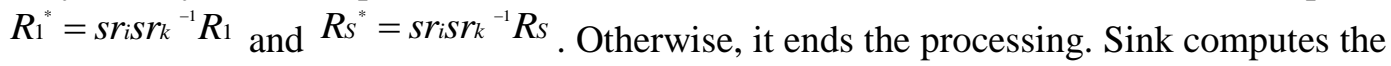
value $\quad V_{3}=H_{3}\left(s_{1} s r \mid A D_{s}, S 3 s r_{k} A D_{\mathcal{O}} M_{k 1}, R_{S}, R_{1}^{*}, T_{2}, V_{1}\right) \quad, \quad$ and sends

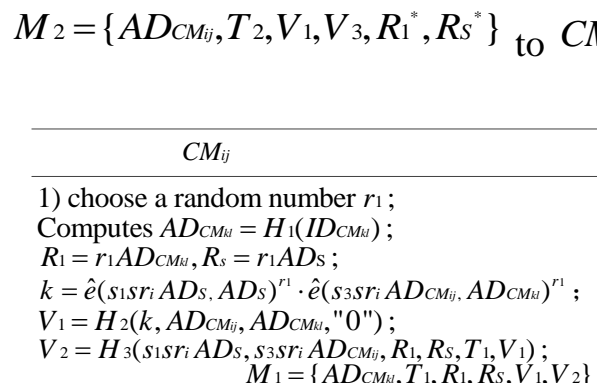

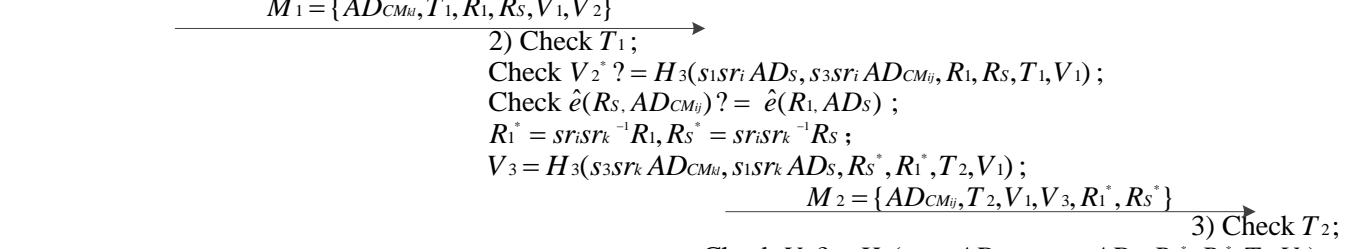
Check $V_{3} ?=H_{3}\left(s_{3} s r_{i} A D_{C M_{k l},} s_{1} s r_{i} A D_{s}, R_{s}{ }^{*}, R_{1}{ }^{*}, T_{2}, V_{1}\right)$; $k^{*}=\hat{e}\left(s_{1} s r_{k} A D_{s}, R_{s}^{*}\right) \cdot \hat{e}\left(s_{3} s r_{k} A D_{C M_{k} l}, R_{1}^{*}\right)$; Check $V_{1}$ ? = $H_{2}\left(k^{*}, A D_{C M_{i j}}, A D_{C M_{k}}, " 0 "\right)$; $s k^{*}=H_{2}\left(k^{*}, A D_{C M_{k},}, A D_{C M_{i j}}, " k e y "\right)$ and store $s k^{*}$; $M_{3}=\left\{A D_{C M_{i j}}, T_{3}, V_{4}\right\}$ Compute $V_{4}=H_{2}\left(k^{*}, A D_{C M k}, A D_{C M_{i j}}, T_{3}\right)$;

5)Check $\overleftarrow{T}_{3}$;

Check $V_{4}$ ? $=H_{2}\left(k, A D_{C M_{k l}}, A D_{C M_{i j}}, T_{3}\right)$;

$s k=H_{2}\left(k, A D_{C M_{k}}, A D_{C M_{i j}}, " k e y "\right)$ and store $s k$;

Figure 6. Authentication and Key Agreement Phase in our Scheme 
Step A3. After received the data package $M_{2}, C M_{k l}$ checks the validity of the timestamp $T_{2}$. If it has grown stale, $C M_{k l}$ ends the session. Otherwise, it judges whether the verifier $V_{3}$ is equal to the hash value $H_{3}\left(s_{1} s r_{k} A D_{s}, s_{3} s r_{k} A D_{C M_{k}}, R_{s}{ }^{*}, R_{1}{ }^{*}, T_{2}, V_{1}\right)$. Only if they are equal, $C M_{k l}$ computes the temporary key $k^{*}$ as follows.

$$
k^{*}=\hat{e}\left(s_{1} s r_{k} A D_{S}, R_{s}{ }^{*}\right) \cdot \hat{e}\left(s_{3} S_{k} A D_{C M_{k},}, R_{1}{ }^{*}\right) \text {. }
$$

Otherwise, it ends the processing. Then, $C M_{k l}$ checks whether the received value $V_{1}$ is equal to the hash value $H_{2}\left(k^{*}, A D_{C M_{i j}}, A D_{C M_{k},}, " 0^{\prime \prime}\right)$. Only if they are equal, $C M_{k l}$ authenticates $C M_{i j}$ and assures the temporary key $k^{*}$. Then, $C M_{k l}$ computes $s k^{*}=H_{2}\left(k^{*}, A D_{C M_{k}}, A D_{C M_{i j}}, " k e y "\right)$, and a value $V_{4}=H_{2}\left(k^{*}, A D_{M_{k l}}, A D_{M},{ }_{3} T\right.$. Finally, it sends $M_{3}=\left\{A D_{C M_{k},}, T_{3}, V_{4}\right\}$ to $C M_{i j}$. Here, $T_{3}$ is a current timestamp.

Step A4. After received the data package $M_{3}, C M_{i j}$ checks the validity of the timestamp $T_{3}$. If it is invalid, it terminates the processing. Otherwise, $C M$ computes a hash value $V_{4}{ }^{*}=H_{2}\left(k, A D_{C M_{k} l}, A D_{C M_{i j}}, T_{3}\right)$. Only if $V_{3}=V_{3}{ }^{*}, C M_{i j}$ conputes the session key $s k$ is equal to $H_{2}\left(k, A D_{C M_{k}}, A_{C M_{i j}}, " k e y "\right)$ with $C M_{k l}$

\section{Correctness and Security Analysis}

In this section, we present the correctness of our improved scheme. Then, we analyze our enhancement scheme regarding security and overcome the weaknesses analyzed in Section 3.

\subsection{Correctness}

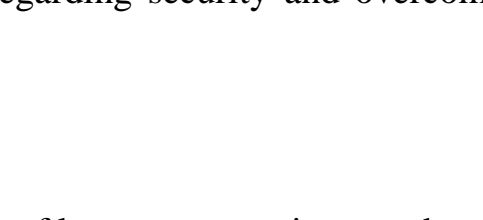

We verify the correctness of key agreement in our scheme as follows.

$$
\begin{aligned}
& k=\hat{e}\left(S_{1 S \hat{n}_{i}} A D_{s}, A D s\right)^{r} \hat{e}\left(s_{3} S r_{i} A D_{C M_{i j}}, A D_{\left.C M_{k}\right)^{r}}\right. \\
& \text { e }\left(s_{1 S r_{i}} A D_{S} r_{1} A D_{S}\right) \cdot \hat{e}\left(s_{3} S r_{i} A D_{C M_{k}}, r_{1} A D_{C M_{i j}}\right) \\
& =\hat{e}\left(s_{1} s r_{i} s_{k} A_{A} D_{s}, s r_{k}^{-1} R S\right) \cdot \hat{e}\left(s_{3} s r_{i} s r_{k} A D C M_{k}, s r_{k}^{-1} R_{1}\right) \\
& =\hat{e}\left(1 s r_{k} A D_{s}, s r_{i} s r_{k}^{-1} R s\right) \cdot \hat{e}\left(s 3 s r_{k} A D_{C M_{k}}, s r_{i} s r_{k}^{-1} R_{1}\right) \\
& =\left(\hat{e}\left(s_{3} s r_{k} A D_{s}, R_{s}{ }^{*}\right) \cdot \hat{e}\left(s_{3} s r_{k} A D_{C M_{k},} R_{1}^{*}\right)\right. \\
& \theta k^{*}
\end{aligned}
$$

The session key, computed by $C M_{i j}$ and $C M_{k l}, s k=H_{2}\left(k, A D_{C M_{k}}, A D_{C M_{i j}}, " k e y "\right)$ and $s k^{*}=H_{2}\left(k^{*}, A D_{C M_{k}}, A D_{C M_{i j}}, " k e y "\right)$ are equal.

\subsection{Security Analysis}

\subsubsection{Authentication}

$C M_{i j}$ sends the data packages $M_{1}=\left\{A D_{C M_{k}}, T_{1}, R_{1}, R_{s}, V_{1}, V_{2}\right\}_{\text {to }}$ tink. Here, the value $V_{2}=H_{2}\left(s_{1} S r_{i} A D_{s}, S 3 s r_{i} A D_{C M_{i j}}, R_{1}, R s, T_{1}, V_{1}\right)$. Without the private keys $s_{1} s r_{i} A D_{s}$ and $S_{3} S r_{i} A D_{C M_{i}}$, the adversary cannot generate a legal data package $M_{1}$ due to the nature of the hash function. Therefore, Sink could authenticate $C M^{i j}$ by checking the correctness of the value $V_{2}$. Similarly, $C M_{k l}$ could authenticate Sink by judging the accuracy of the $V_{3}$. A sensor node $C M_{k l}$ sends the message $M_{3}=\left\{A D_{C M_{k},}, T_{3}, V_{4}\right\}$ to another node $C M_{i j}$. 
Here, the value $V_{4}=H_{2}\left(k^{*}, A D_{C M_{k}}, A D_{C M_{i j}}, T_{3}\right)$. Since only $C M_{i j}$ and $C M_{k l}$ can compute the temporary key $k^{*}$. Therefore, $C M_{i j}$ could authenticate $C M_{k l}$ through checking the correctness of the $V_{4}$. It is essential to note that the scheme should ensure adequate entropy of the keys in the System Parameter Generation Phase in order to prevent the offline guessing attack.

\subsubsection{Security Key Establishment}

Key security is a critical requirement for a key agreement scheme. Assume that the

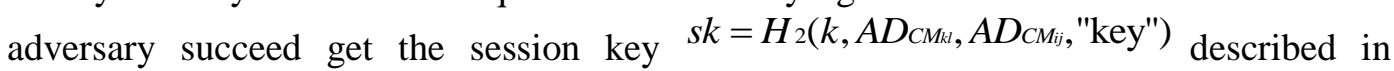
Section 5. Since the hash function has the one-way characteristic, the adversary should obtain the temporary $k$ or $k^{*}$ for computing the key $s k$. However, the temporary key $k$ is established by $C M_{i j}$ in the Step A1. Since the adversary does not know the private values of $C M_{i j}$, he/she cannot compute the temporary key $k=\hat{e}\left(s_{1} S r_{i} A D s, A D S\right)^{r_{1}} \cdot \hat{e}\left(s_{3} S r_{i} A D_{C M_{i j}}, A D_{C M_{k}}\right)^{r_{1}}$. Moreover, In the Step A3, the temporary $k^{*}$ is established by $C M_{k l}$. Since the adversary does not know the values of $s_{1} S r_{k} A D_{S}$ and $s_{1} S r_{k} A D C M_{k l}$ together, he/she cannor calculate the temporary key $k^{*}$ by formula $k^{*}=\hat{e}\left(s_{1} s r_{k} A D_{s}, R^{*}\right) \cdot \hat{e}\left(s_{3} S r_{k} A D_{C M_{k},}, R_{1}^{*}\right)$

Our scheme also achieves perfect forward secrecy. Since the numbers of $R_{1}$ and $R s$ are randomly computed in every commutuication, the values of $R_{1}^{*}$ and $R_{s}{ }^{*}$ are freshly in every stage. Therefore, all the historicalsession keys will still secure even if the long-term private keys are disclosed in future. Base on the one-way function and the freshness of $R_{1}$ and $R s$, the adversary cannot obtan any infomation about the future session key even if the current session key $s k$ or $s k$ is compromised.

\subsubsection{Security Against Replay Attacks}

Our scheme can resist replay attack because we used the timestamps. If an adversary replays the eavesdropped message, then the sensor nodes will detect the attack when examining the current time During the authentication and key agreement phase, when Sink receives a data package $M_{1}=\left\{A D_{C M_{k},}, T_{1}, R_{1}, R_{s}, V_{1}, V_{2}\right\}$, it verifies the timestamp $T_{1}$ with the current time. If the message is a replay message, then Sink will find it. If adversary change the timestamp $T_{1}$ in data package $M_{1}$, however, it cannot know the values of $S_{1} S r_{i} A D_{s}, S_{1} S r_{i} A D_{C M_{i j}}$, Sink will find the replay when it check the value $V_{2}$. Similarly, when $C M_{k l}$ receives the data package $M_{2}=\left\{A D_{C M_{i j}}, T_{2}, V_{1}, V_{3}, R_{1}{ }^{*}, R_{s}{ }^{*}\right\}$, it judges the timestamp $T_{2}$ to against the message replay. Similarly, when $C M_{i j}$ receives the data package $M_{3}=\left\{A D_{C M_{k},} T_{3}, V_{4}\right\}$, it resist the message replay by judging the timestamp $T_{3}$ and the session key $s k$. It is should admit that the synchronization problem is the main vulnerability to timestamp, However, the situation has been released with the development of the sensor technologies [26] such as using the linear regression to achieve long-term synchronization and using the time base signal in the Global Position System (GPS). The timestamp is the economic and effective means to resist the replay attack in WSN. 


\subsubsection{Security Against Insider Attacks}

In the proposed scheme, Sink computes and distributes different private keys $\left(S_{1} S r_{i} A D_{s}, S_{2} S r_{i} A D_{C H}, S_{3} S r_{i}\right)$ for various CHs. Thus, the adversary $\mathrm{CH}$ cannot get the information including the private keys of the other CHs. Furthermore, if we assume that an adversary CM who has registered as $C M_{i j}$ can obtain $S_{1} S r_{i} A D_{s}$ and $S 3 S r_{i} A D_{C M_{i}}$ from $\mathrm{CH}_{i}$. However, he/she cannot calculate ${ }^{S_{3} S r_{i}}$ from ${ }^{S}{ }_{3} r_{i} A D_{C M_{i j}}$ except he/she can solve the DLP assumption. Thus, our scheme destructs the attack conditions as Step H1 and Step M1. As a result, the scheme prevents the adversary to generate the temporary key $k$ and the session key $s k$ in sequence. Therefore, the proposal could withstand the insider attack. It should be noted that Sink has an enough computing power as a data concentrator node. Although our proposal increases the computation cost of Sink, the advice is feasible to WSN.

\subsubsection{Security Against Impersonation Attacks}

To impersonate $C M_{i j}$ to Sink, an adversary has to generate a legal message $M_{1}=\left\{A D_{C M_{k}}, T_{1}, R_{1}, R_{s}, V_{1}, V_{2}\right\}$, where $V_{2}=H_{2}\left(s_{1 S r_{i}} A D_{s}, S_{3 s} A_{i} A D_{C M_{i j}}, R_{1} R_{S}, T_{1}, V_{1}\right)$. The adversary cannot compute $V_{2}$ because the values $S S_{A} A D_{s}$ and $S_{3} S_{i} A D_{C M_{i}}$ are private key of $C M_{i j}$. Therefore, the adversary cannot impersonate $C M_{i}$ to Sink. To attack Sink to $C M_{k l}$, an adversary has to generate a data $M_{2}=\left\{A D C M_{i j} T_{2}, V_{1}, V_{3}, R{ }^{*}, R s^{*}\right\}$, where $V_{3}=H_{3}\left(s_{1} s r_{k} A D_{s}, S_{3} s r_{k} A D_{C M_{k}}, R_{s}{ }^{*}, R_{1}^{*}, T_{2}, V_{1}\right)$ Without the knowledge of $s_{1} s r_{k} A D_{s}$ and $S_{3} S r_{k} A D_{C M k}$, an adversary cannot generate $V_{3}$. Thus, the adversary cannot impersonate Sink to $C M_{k l}$. To impersonate $C M_{k}$ to $C M_{i j}$, an adversary has to generate a legal message $M_{3}=\left\{A D_{C M_{k}} T_{3}, V_{4}\right\}$. Here the authentication value $V_{4}=H_{2}\left(k^{*}, A D_{C M_{k}}, A D_{C M_{i}} T_{3}\right)$. The adversary cannot compute $V_{4}$ since he/she does not know the temporary key . Therefore, the adversary cannot impersonate $C M_{i j}$ to $C M_{k l}$.

\subsection{Security Comparison}

We compared the propesed scheme with Lee and Kim's scheme and Guo et al.'s scheme in terms of security properties. Similar to the Kim's work, P1, P2, P3, P4, P5 and P6 denote the key agceement, the authentication, the impersonation attack, the key freshness, the replay attack and the insider attack, separately. According to Table 2, we can conclude that the proposed scheme delivers a higher level of security compared to related wôrks. 
Table 2. The Security Comparison

\begin{tabular}{l|cccc}
\hline \hline & Ours & $\begin{array}{c}\text { Lee and Kim's } \\
{[7]}\end{array}$ & Kim's[24] & Guo et al.'s [8] \\
\hline P1 & Provide & Provide & Provide & Provide \\
P2 & Provide & Provide & Provide & Provide \\
P3 & Resistance & Resistance & Resistance & Resistance \\
P4 & Resistance & Provide & N/A & N/A \\
P5 & Resistance & N/A & N/A & N/A \\
P6 & Resistance & N/A & N/A & N/A \\
\hline \hline
\end{tabular}

\section{Conclusion}

We discuss several security weaknesses in the paper under a new security model. There is an adversary who can legally get the private parameter of $\mathrm{CM}$ or $\mathrm{CH}$ and control the channel between two CMs in the attack model. After that, we point out the two weaknesses of Lee and Kim's scheme [7] in our model. Then we propose an enhanced scheme based on the bilinear pair to overcome these weaknesses. Our proposal increases its security strength by using the Sink nodes. The forthcoming work is to achieve a formal proof method of the key agreement scheme in WSN. Then we achieve it in the real sensor hardware environments.

\section{Acknowledgements}

The authors would like to thank the anonymous reviejws for their valuable comments. This paper is supported by the Nat onal Key Basic Research Program (973 program) through project 2012CB315905, by the Natural Science Foundation through projects 61370190, 61272501, 61173154,61402029@and 61003214, and by the Beijing Natural Science Foundation through projects 4132056 and 4122041 . The authors also thank China Aeronautical Radio Electonjics Reseateh Institute for its funding.

\section{References}

[1] A. Perrig R Szewezyk, J. D. Tygar, V. Wen and D. E. Culler, "SPINS: Security protocols for sensor networks", Wireless Networks, vol. 8, no. 5, (2002), pp. 521-534.

[2] I. F. Akyildiz, W. Su, Y. Sankarasubramaniam and E. Cayirci, "A survey on sensor networks", IEEE Communications magazine vol. 40, no. 8, (2002), pp. 102-114.

[3] L. Y. Li and C. D Liu, "An improved algorithm of LEACH routing protocol in wireless sensor networks", Journa oH Harbin University of Science and Technology, vol. 20, no. 2, (2015), pp. 75-79.

[4] A. Perrig, J. Stankovic and D. Wagner, "Security in wireless sensor networks", Communication of the ACM, vol. 47, ho. 6, (2004), pp. 53-57.

[5] R. D. Pietro, L. V. Mancini and S. Jajodia, "Providing secrecy in key management protocols for large wireless sensors networks", Ad Hoc Networks, vol. 1, no. 4, (2003), pp. 455-468.

[6] C. Y.Chen and H. C. Chao, "A survey of key distribution in wireless sensor networks", Security and Communication Networks, vol. 7, no. 12, (2014), pp. 2495-2508.

[7] S. Lee and H. Kim, "Freshness Consideration of Hierarchical Key Agreement Protocol in WSNs", International Journal of Security and Its Applications, vol. 8, no. 1, (2014), pp. 81-91.

[8] H. Guo, Y. Mu, Z. Li and X. Zhang, "An efficient and non-interactive hierarchical key agreement protocol”, Computers \& Security, vol. 30, no. 1, (2011), pp. 28-34.

[9] X. He, M. Niedermeier and H. De Meer, "Dynamic key management in wireless sensor networks: A survey", Journal of Network and Computer Applications, vol. 36, no. 2, (2013), pp. 611-622.

[10] O. K. Sahingoz, "Large scale wireless sensor networks with multi-level dynamic key management scheme", Journal of Systems Architecture, vol. 59, no. 9, (2013), pp. 801-807.

[11] R. Watro, D. Kong, S. Cuti, C. Gardiner, C. Lynn and P. Kruus, "TinyPK: securing sensor networks with public key technology", Proceedings of the 2nd ACM workshop on Security of ad hoc and sensor networks, Washington, DC, USA, (2004), pp. 56-64. 
[12] G. Gaubatz, J. Kaps and B. Sunar, "Public key cryptography in sensor networks-revisited", in Security in Ad-hoc and Sensor Networks, Edited C. Castelluccia, H. Hartenstein, C. Paar and D. Westhoff, Springer, Heidelberg, vol. 3313, (2005), pp. 2-18.

[13] W. Du, R. Wang and P. Ning, "An efficient scheme for authenticating public keys in sensor networks", Proceedings of the 6th ACM international symposium on Mobile ad hoc networking and computing, Urbana-Champaign, IL, USA, (2005), pp. 58-67.

[14] C. P. Gouvêa, L. B. Oliveira and J. López, "Efficient software implementation of public-key cryptography on sensor networks using the MSP430X microcontroller", Journal of Cryptographic Engineering, vol. 2, no. 1, (2012), pp. 19-29.

[15] W. Shi and P. Gong, "A new user authentication protocol for wireless sensor networks using elliptic curves cryptography”, International Journal of Distributed Sensor Networks, vol. 2013, no. 730831, (2013), pp. 1-7.

[16] A. Liu and P. Ning, "TinyECC: A configurable library for elliptic curve cryptography in wireless sensor networks", International Conference on Information Processing in Sensor Networks 2008, St. Louis, MO, USA, (2008), pp. 245-256.

[17] N. Gura, A. Patel, A. Wander, H. Eberle and S. C. Shantz, "Comparing elliptic curve cryptography and RSA on 8-bit CPUs", Proceedings of the 6th International Workshop Cambridge, MA, USA, (2004), pp. 119-132.

[18] A. Shamir, "Identity-based cryptosystems and signature schemes", in Advances in Cryptology Edited G. R. Blakley and D. Chaum, Springer, Heidelberg, vol. 196, (1985), pp. 47-53.

[19] D. Boneh and M. Franklin, "Identity-based encryption from the Weil pairing", SIAM Journal on Computing, vol. 32, no. 3, (2003), pp. 586-615.

[20] L. Chen and C. Kudla, "Identity based authenticated key agreement protocols from pairings", Proceedings on the 16th Computer Security Foundations Workshop, Pacific Grove, CA, USA, (2003), pp. 219-233.

[21] L. B. Oliveira, D. F. Aranha, C. P. Gouvêa, M. Scott, D. F. Câmara, J. Lópezand R. Dahab, "TinyPBC: Pairings for authenticated identity-based non-interactive key distribution in sensor networks", Computer Communications, vol. 34, no. 3, (2011), pp. 485-493.

[22] S. Sung and J. Ryou, "ID-based sensor node authentieation for multi-layer sensor networks", Journal of Communications and Networks, vol. 16, no. 4, (2014), pp. 363-370.

[23] L. B. Oliveira and R. Dahab. "Pairing-based cryptography fo sensor networks", Proceedings of the 5th IEEE International Symposium on Network Computing and Applications, Cambridge, MA, USA, (2006).

[24] H. Kim, "Efficient and non-interactive hierarchical key agreement in WSNs", International Journal of Security and Its Applications, vol 7 , no. 1, (2013), pp. 159-170.

[25] H. Lu, J. Li and M. Guizani, "Secure and efficient data transmission for cluster-based wireless sensor networks", IEEE Transadions on Parallelánd Distributed Systems, vol. 25, no. 3, (2014), pp. 750-761.

[26] J. J. P. Solano and S. F. Castell, "Adaptive time window linear regression algorithm for accurate time synchronization in wireless sensor networks", Ad Hoc Networks, vol. 24, no. A, (2015), pp. 92-108.

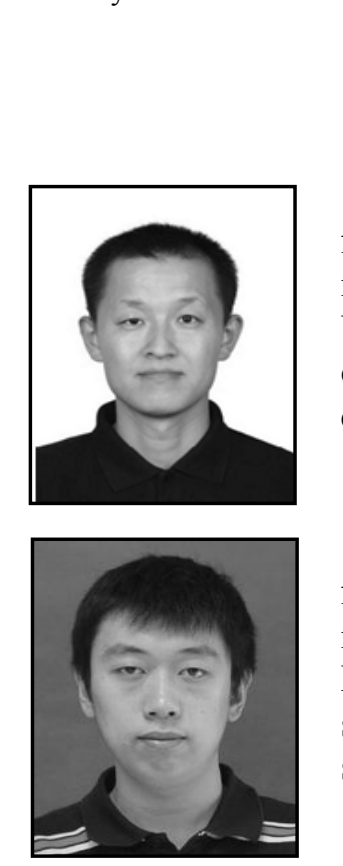

\section{Authors}

Kefei Mao, He received the M.Sc. degree in computer science from the University of Chinese Academy of Sciences, Beijing, China, in 2007. He is currently pursuing the doctoral degree with Beihang University, Beijing, China. His current research interests include optimization algorithms design and analysis, computational complexity, and network schemes.

Jie Chen, He received the M.Sc. degree in Electronic engineering from the Northwest Polytechnic University, Xi'an, China, in 2010. He is currently pursuing the doctoral degree with Beihang University, Beijing, China. His current research interests include Ad hoc network security, Future Network Design and Software Define Network security. 


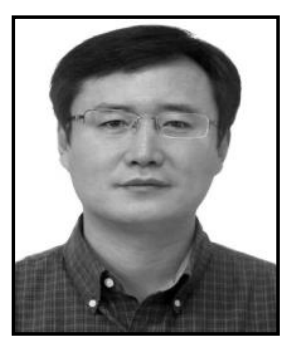

Jianwei Liu, He received the B.S. and M.S. degrees in Electronic and Information from Shandong University, Shandong, China in 1985 and 1988, respectively. He received his Ph.D. degree in Communication and Electronic System from Xidian University, Shanxi, China in 1998. Now, he is a Professor of Electronic and Information Engineering at Beihang University, Beijing, China. His current research interests include wireless communication network, coding theory, and information security.

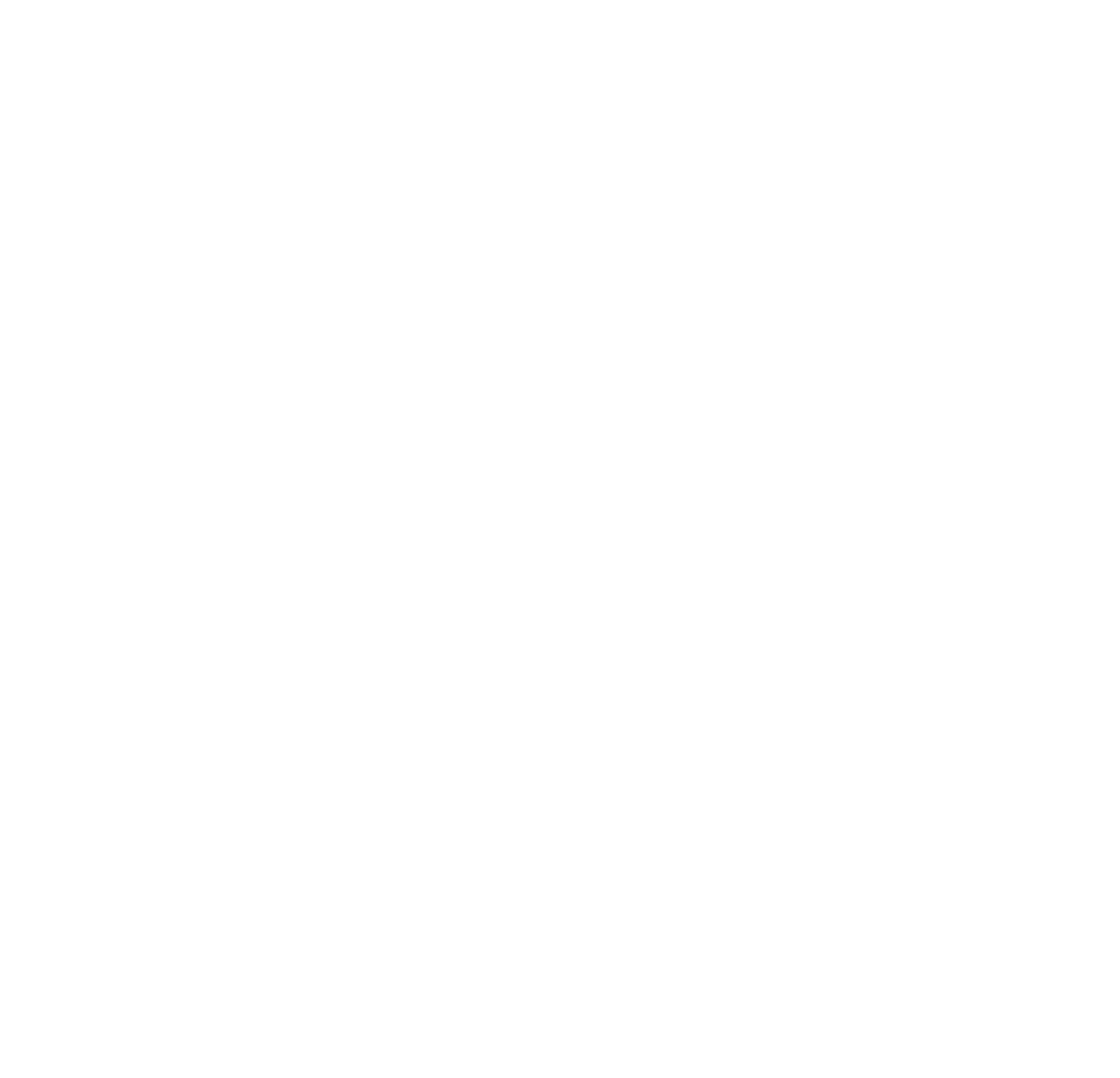


International Journal of Multimedia and Ubiquitous Engineering

Vol.11, No.9 (2016)

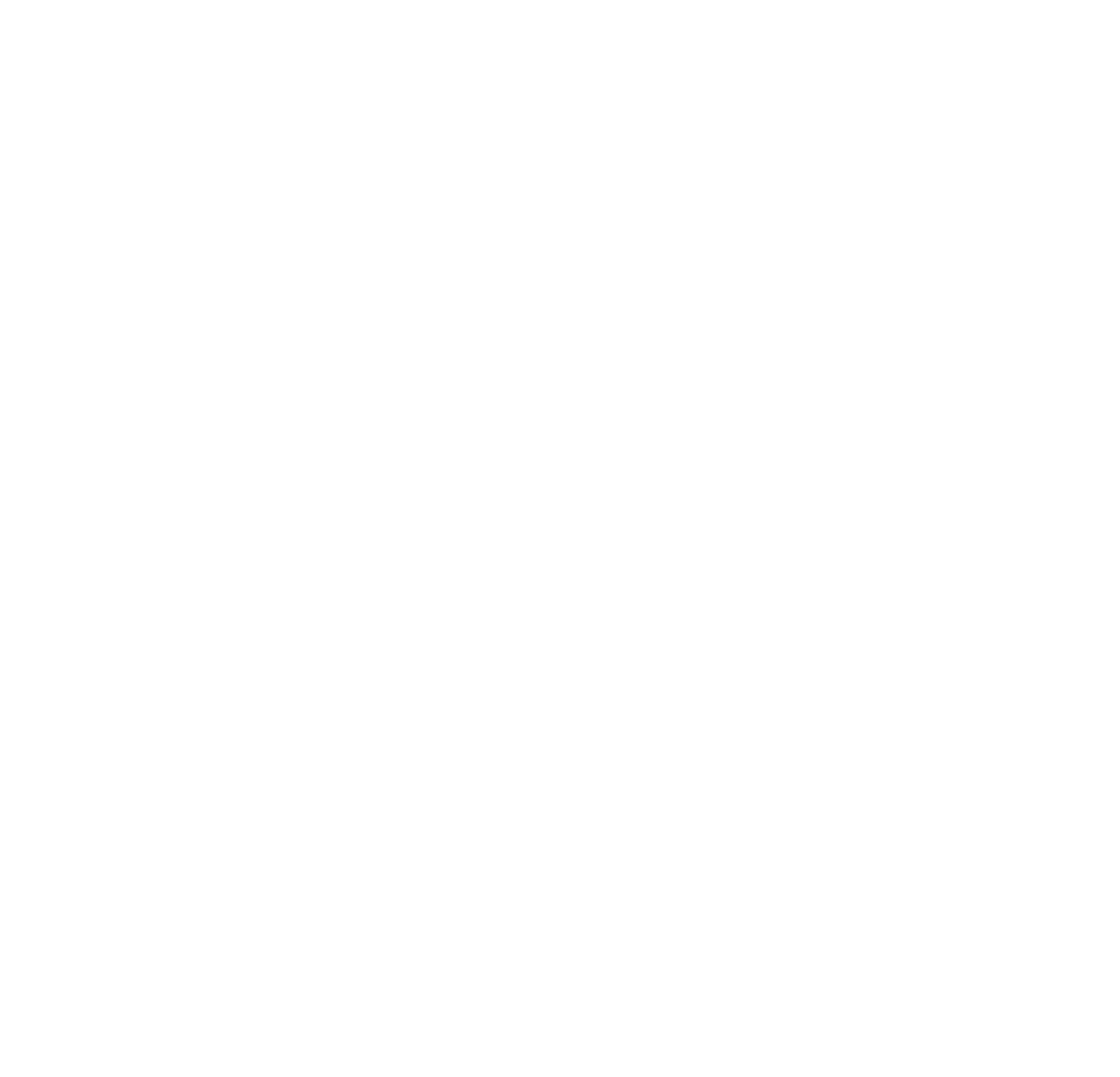

